\title{
Using QR Code for Uniform Representation of Content in Cross-Border Exchange of ePrescriptions in the EU
}

\author{
Evgeniy KRASTEV a1, Petko KOVACHEV a , Dimitar TCHARAKTCHIEV ${ }^{\text {b }}$ and \\ Simeon ABANOS ${ }^{\mathrm{a}}$ \\ ${ }^{a}$ Faculty of Mathematics and Informatics, Sofia University St. Kliment Ohridsky, \\ Bulgaria \\ ${ }^{\mathrm{b}}$ Department of Medical Informatics, Medical University, Sofia, Bulgaria
}

\begin{abstract}
This paper proposes an approach and demonstrates its application for cross-border exchange of ePrescriptions in the European Union. A business process model of the main use case for exchange of prescription content in the eHealth Digital Service Infrastructure is created and analyzed. The novelty in this approach is the proposed encoding of the basic dataset in a Quick Response $(\mathrm{QR})$ code in terms of an XML scheme that is independent of clinical models or proprietary database structures. It allows to inverse the dataflow control in the chain of message exchanges between Dispenser and National Contact Points. The proposed inversion of control positions the citizen with the QR code of the prescription in the center of that chain of message exchanges between the main actors of the business process. The independent format of content representation in the QR code allows the actors in the message exchange to auto-populate data in their registers when the medicine is dispensed. Initial results are reported and reveal the advantages of embedding prescription details in QR code employing a common independent XML scheme.
\end{abstract}

Keywords. ePrescription, QR code, inversion of control, cross-border exchange, health informatics, XML, business process model and notation

\section{Introduction}

The prescription of medicinal products in electronic format (ePrescription) is a common practice in many countries like the Unites States (Surescripts [1]), Canada (PrescribeIT [2]) or Australia (eRx [3]). Sweden and Denmark are among the first EU member states that have reported nationwide implementation of ePrescription services early in 2010 [4]. The benefits of introducing ePrescription services have increased substantially with the advance of information technologies. Therefore, the number of countries that have deployed such services and joined the eHealth Digital Service Infrastructure(eHDSI) [5] has rapidly increased. The major benefits for the patients using ePrescriptions include improving healthcare quality by minimization of medication-prescribing errors, reduced transcription errors in serving the prescribed medicine at the pharmacy due to handwriting or incomplete data in paper prescriptions. The use of ePrescriptions means a reduction in the number of visits to clinics just for prescribing a medicine. It allows to

1 Corresponding Author: Evgeniy Krastev, Faculty of Mathematics and Informatics, Sofia University "St. Kliment Ohridsky", 5 James Bouchier blvd., Sofia, Bulgaria; E-mail: eck@fmi.uni-sofia.bg. 
avoid the risk of being exposed to a virus, which is of essential importance for patients suffering chronic diseases or under quarantine in the current COVID-19 pandemic. The mission of eHDSI is to deploy services for ePrescriptions exchange at EU level.

The greatest challenge is the achievement of interoperability between different software architectures and heterogeneous clinical information models HL7 [6], openEHR [7][8] or other proprietary models in nationwide implementations of ePrescription services. The objective of this study is to propose an approach for crossborder exchange of ePrescription content based on uniform representation of the eHDSI basic dataset in QR code. The following section presents the methods employed to achieve this objective. Section 3 reports experimental results and the conclusions are outlined in Section 4.

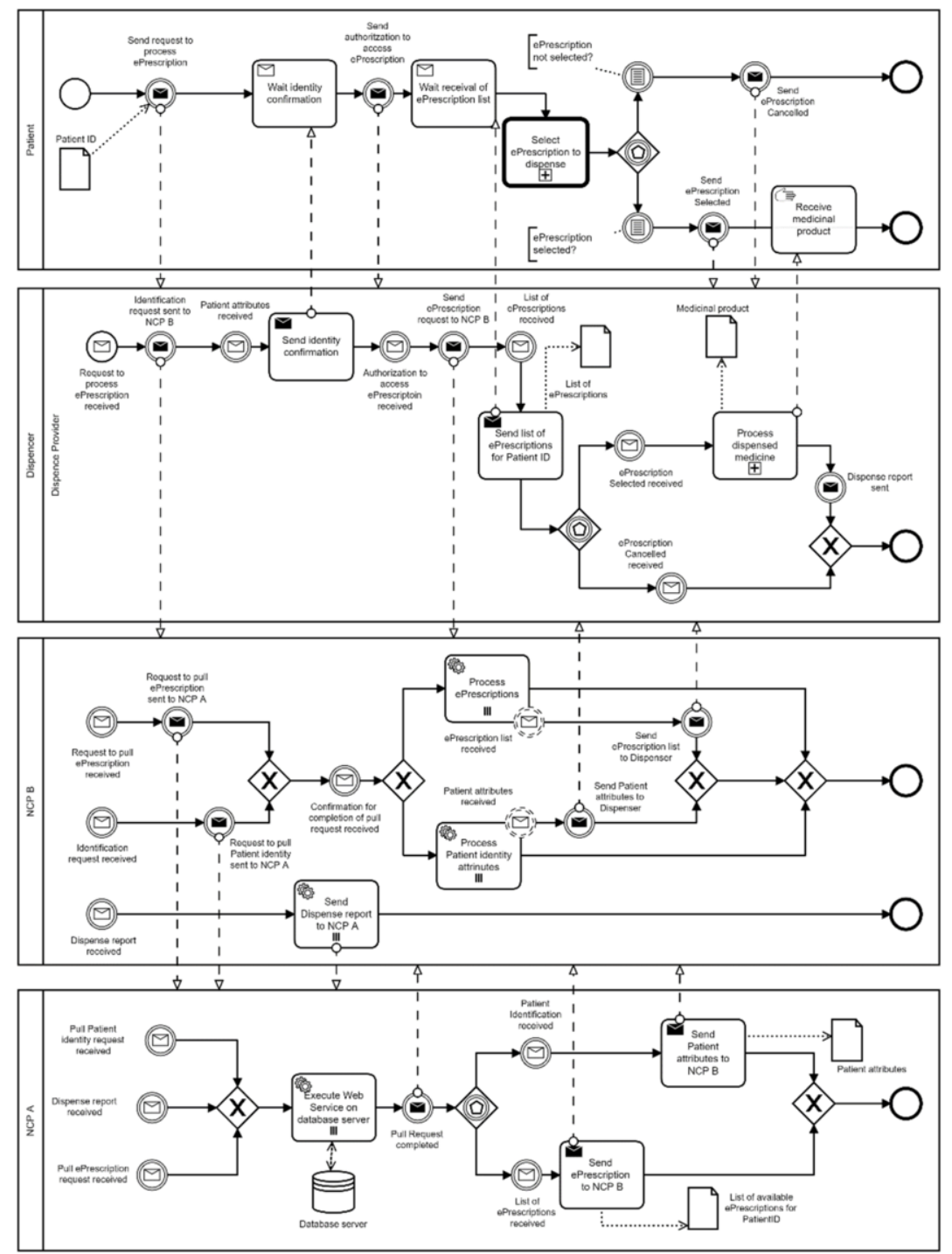

Figure 1. Business process model of use case "Medicine already prescribed in country A". 


\section{Methods}

There are four use cases for the implementation of the ePrescription service in eHDSI at EU level [5]. In the existing literature these use cases are not analyzed as business processes. For better understanding of the main use case we have designed a Business Process and Model Notation (BPMN) diagram ( Figure 1) of the business process (BP). The notations and their usage in this diagram follow the BPMN standard [9]. The main actors are the Patient, Dispenser(Dispenser Provider), National Contact Points (NCP) in countries A and B. It employs a centralized architecture for medicine dispensing during the stay of Patient in country B, where the prescription is issued and stored in country A and NCP A accordingly. Each ePrescription refers to a single medicinal product.

The analysis of the BP identifies several requirements that are difficult to implement in practice. The Patient request for receive the medicine prescribed in country A triggers a chain of message exchanges between the Dispenser, NCP B and NCP A. It assumes availability of servers and reliable network connections between these actors. Most pharmacies (Dispenser) will not be able to satisfy this requirement or at least it will be time-consuming to execute. On the other side the communication between NCP A and NCP B requires to map bidirectionally prescription details employed in both A and B in their Clinical Information Model(CIM). For example, some CIM use HL7 templates or resources, while other use approved EU standards like CEN 13606 or openEHR specifications. Therefore, eHDSI requires NCPs to store prescription details in so-called eHDSI "friendly" format of a basic dataset. The use case execution is full of challenges and currently eHDSI allows interoperability among systems just at the technical level.

In this paper we propose to inverse the control over the prescription details by representing them in Quick Response $(\mathrm{QR})$ code employing independent XML scheme readable at all pharmacy locations. It allows auto-population of data in the specific CIM format for NCP B and NCP A at the time of medicine request or later, when offline.

\section{Results}

The practicability of the proposed approach has been evaluated in a web application [10] where the user interface allows to generate the basic dataset of an eHDSI "friendly" ePrescription. For this purpose we have designed a XML scheme (Figure 2) that includes all the data elements as they are structured and described in the latest version of the ePrescription service by eHDSI [5]. In close relation to the existing practice and standards in health informatics, we allow to encode the product code in a selected codesystem (ATC, RxNorm etc.) and to add more than one active ingredient per medicinal product (code, strength, units). For example, knowing the active ingredients of a medicinal product it can be replaced by another product having the same description of the active ingredients, but known under a different name in the country of dispensing.

Once all the required data is recorded, the web application generates the prescription content in XML format that is validated against the XML scheme of the ePrescription. The QR code of the prescription content is shown in Figure 3 in its original size, where the data elements are transformed in JSON format by a RESTful web service. Thus, the QR code can be scanned by a digital device like a smartphone or sent to the Patient by email. Having the prescription in QR code a Patient can inverse the control of events in the BP shown in Figure 1 by initiating it even at remote locations, where the transactions between Dispenser, NCP B and NCP A can be completed off-line making use of 
communication protocols for secure payments. Similarly to its usage in secure payment applications a QR code can ensure prescription data authenticity, integrity and nonrepudiation by employing computer security tools like digital signatures and encryption. Besides, the adoption of the ISO Identification of Medicinal Products standards will reduce significantly the time doctors spend on compiling ePrescriptions with this application.

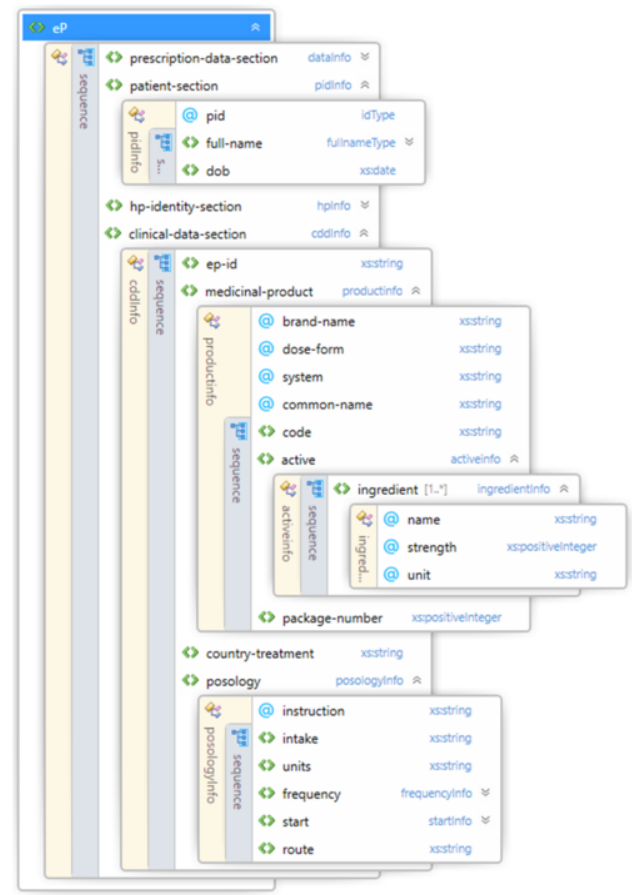

Figure 2. XML scheme of the eHDSI basic data set in an ePrescription.

\section{Conclusion}

The business process of the main eHDSI use case in cross-border exchange of ePrescriptions is analyzed and problems in its practical implementation are identified. This paper proposes an approach to inverse the control over the prescription details in this use case by employing QR codes for uniform representation of ePrescription content in standard XML format. The content structure is validated against an XML scheme designed to match the eHDSI basic dataset. The XML scheme is independent of any CIM and enables exchange of prescription content between data storages of potentially different CIM in each EU country. This way, one of the major challenges in cross-border exchange is resolved. QR codes enable offline processing of prescriptions at remote locations by employing technologies used for secure payments and border pass control. Besides, QR codes allow prescription data to be auto-populated into pharmacy registers. A fully functional software prototype is developed for evaluating this approach in practice. Initial results are reported and reveal the advantages of embedding CIM independent prescription details in $\mathrm{QR}$ code. Future research work plans include 
evaluation of the application usability in a real-life environment by implementing computer services for ensuring secure processing of ePrescription content in QR code.

\section{Acknowledgement}

This research is supported by the National Scientific Program eHealth in Bulgaria.

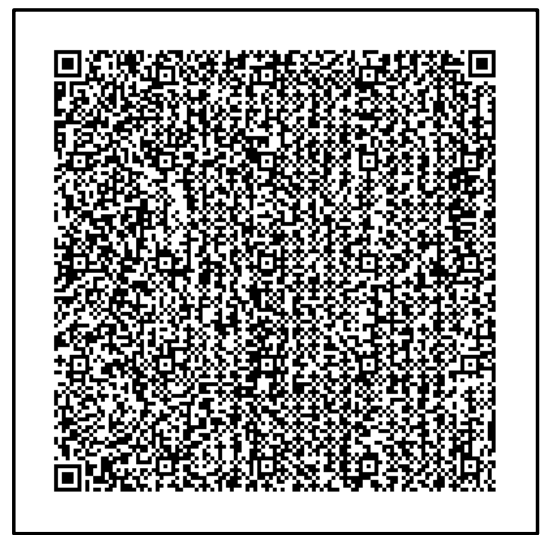

Figure 3. Sample QR code of ePrescription with JSON structured eHDSI content.

\section{References}

[1] Surescripts., The Nations's Most Trusted and Capable Health Information Network. 2021, Available at https://surescripts.com/, Accessed January, 2021.

[2] Canada Health Infoway. Infoway- The PrescribeIt Advantage. 2021. Available at https://prescribeit.ca/component/edocman/198-the-prescribeit-advantage/download?Itemid=106.

[3] eRx. ePrescribing. Rx Script Exchange. 2020, Available at https:/www.erx.com.au/eprescribing/. Accessed January, 2021.

[4] Kierkegaard P. E-Prescription across Europe. Health and Technology. 2013;3:205-219.

[5] eHDSI Business Analyst. eP/eD Use Case. 17 September 2020. Available at https://ec.europa.eu/cefdigital/wiki/pages/viewpage.action?pageId=55887070, Accessed January, 2021.

[6] HL7. HL7 7 Products. Master Grid. $2020 . \quad$ Available at http://www.hl7.org/implement/standards/product matrix.cfm, Accessed January, 2021.

[7] openEHR. The EHR Information Model. The openEHR Foundation. December 2020. Available at https://specifications.openehr.org/releases/RM/latest/ehr.html\# the ehr information model.

[8] ISO/TC 215. ISO 13606:2019 Health informatics.Electronic health record communication," ISO, 2019.

[9] Object Management Group, Business Process Model and Notation, OMG, 2021.

[10] Kovachev P. Uniform representation of ePrescription content in QR code. YouTube, 2021. Available at https://www.youtube.com/watch?v=fvOSVpxCefw, Accessed Jan 14, 2021]. 